\title{
Getting R-AEDI to save lives in Singapore
}

\author{
Si Yong Ivan $\underline{C h u a}^{1}$, MBBS, MCEM, Yih Yng $\underline{N g}^{2}$, MBBS, MRCS, Marcus Eng Hock Ong $^{1,3}$, MBBS, FRCS
}

\begin{abstract}
Early cardiopulmonary resuscitation (CPR) and defibrillation prior to the arrival of emergency medical services can improve survival from out-of-hospital cardiac arrest (OHCA) with good neurological outcome. However, the rate of local bystander CPR is only $24.3 \%$ and bystander defibrillation $2.1 \%$. In 2015, the R-AEDI (Registry for AED Integration) initiative was started to improve OHCA survival rates. R-AEDI alerts volunteers to nearby OHCA cases via the myResponder mobile application. In 2015-2017, 7,682 AEDs were mapped and made accessible via this app. Comprehensive site inspections also resulted in fewer non-functional AEDs, as AED owners were educated on the importance of the maintenance of pads and batteries. The AED heat map allows us to identify areas that are lacking in or require improved public access AED coverage. The online AED registry found in the myResponder app is useful to locate AEDs rapidly during OHCAs. More community education would improve the rate of bystander defibrillation.
\end{abstract}

Keywords: automated external defibrillator, bystander, cardiac arrest, public access defibrillator

\section{INTRODUCTION}

Out-of-hospital cardiac arrest (OHCA) is a global health issue that affects around 350,000 Americans $^{(1)}$ and 275,000 Europeans $^{(2)}$ annually. Effective treatment of OHCA hinges on Cummins' 'chain of survival' - immediate recognition of cardiac arrest and activation of the emergency response system, early cardiopulmonary resuscitation (CPR), early defibrillation, and early advanced life support. ${ }^{(3)}$ The medical literature has tended to focus more on hospital and advanced life support treatment rather than community treatment and basic life support. ${ }^{(4)}$ However, there is increasing recognition that early CPR and defibrillation prior to the arrival of emergency medical services (EMS) can help to improve OHCA survival with good neurological outcome. ${ }^{(5,6)}$

The latest American Heart Association statistics revealed that bystander CPR occurred in $43.6 \%$ of OHCA cases in the United States, but automated external defibrillator (AED) usage occurred in only $6.7 \%$ of cases. ${ }^{(1)}$ There was a similar trend in Singapore: bystander CPR occurred in $24.3 \%$ of OHCA cases, while the rate of bystander defibrillation was $2.1 \% .{ }^{(7)}$ A recent systematic review identified key barriers to the use of public access defibrillators (PADs), including problems locating AEDs, poor accessibility, limited availability and lack of information sharing. ${ }^{(8)}$ This paper highlights initiatives that use smart technology to improve the survival rates of OHCA victims in Singapore.

\section{MYRESPONDER APP AND AED REGISTRY}

MyResponder is a local first responder geolocation application that was launched on 17 April 2015. It uses crowdsourcing to increase bystander CPR rates by alerting volunteer first responders who are within $400 \mathrm{~m}$ of an OHCA victim. Anyone in Singapore can become a first responder after registration, simply by entering their local mobile number and NRIC/FIN (National Registration
Identity Card/Foreign Identification Number). The app also shows the location of the nearest AED on the map, allowing others to assist by retrieving the AED. As seen on the app, as of 28 January 2019, there were 39,127 registered responders and 18,871 responses to cases.

At the same time, the Registry for AED Integration (R-AEDI) initiative was developed to improve the management of public AEDs and assist dispatchers and the public to increase AED usage. This collaboration between the Singapore Civil Defence Force (SCDF) and Singapore Heart Foundation (SHF) comprises two key components: (a) alerting volunteers to nearby OHCA cases via the myResponder app; and (b) building a national registry of PADs. Since 10 July 2015, the SHF has been engaged by the Ministry of Home Affairs (MHA) to promote the R-AEDI project. The SHF is responsible for the establishment and maintenance of the national AED registry, with a target of 6,000 AEDs in the registry by the end of 2018 .

With the engagement of the SHF, the locations of all existing AEDs in the registry are verified, audited and updated. The AED's serial number is also recorded, alongside the brand, model and expiry dates of pads and batteries. From 2015 to 2017, 7,682 AEDs were mapped in Singapore (Fig. 1), and the number is expected to increase. A significant proportion of AEDs in R-AEDI are under the SCDF's Save-A-Life initiative, in which AEDs are installed at the lift lobby of every two blocks of flats, since $70.4 \%$ of OHCAs occur in residential locations. ${ }^{(9)}$ To date, 440 private companies have registered their AEDs. Comprehensive site inspections have also found that the number of non-functional AEDs has been decreasing, as AED owners are educated on the importance of maintenance of the pads and batteries, and sent reminders when these are due for replacement.

The local AED map is shown in Fig. 2. As seen on this map, nature reserves and parks, industrial estates, private estates,

${ }^{1}$ Department of Emergency Medicine, Singapore General Hospital, ${ }^{2}$ Department of Emergency Medicine, Tan Tock Seng Hospital, ${ }^{3} \mathrm{Health}$ Services and Systems Research, Duke-NUS Medical School, Singapore

Correspondence: Dr Chua Si Yong Ivan, Consultant, Department of Emergency Medicine, Singapore General Hospital, Outram Road, Singapore 169608. Ivan.chua.s.y@singhealth.com.sg 
farming areas and offshore islands are lacking AEDs. Sensitive areas such as military bases and camps do have AEDs, but they have been omitted from this map due to restricted public access.

\section{NEED FOR AN AED REGISTRY}

Despite the life-saving potential of AEDs, their effectiveness is time-dependent, as ventricular fibrillation will become refractory to defibrillation, eventually degenerating into asystole. The probability of survival rapidly declines by $7 \%-10 \%$ with every minute of delay in defibrillation. ${ }^{(10)}$ To date, AEDs are not capable of broadcasting location or status data electronically. Thus, an accurate, easily accessible AED map can help people to locate them in an emergency, either directly through smartphone applications or through communication with EMS. ${ }^{(11)}$

Over the years, some private and public organisations installed AEDs in various locations, but as there was no national AED registry prior to 2013, the public and the SCDF 995 control

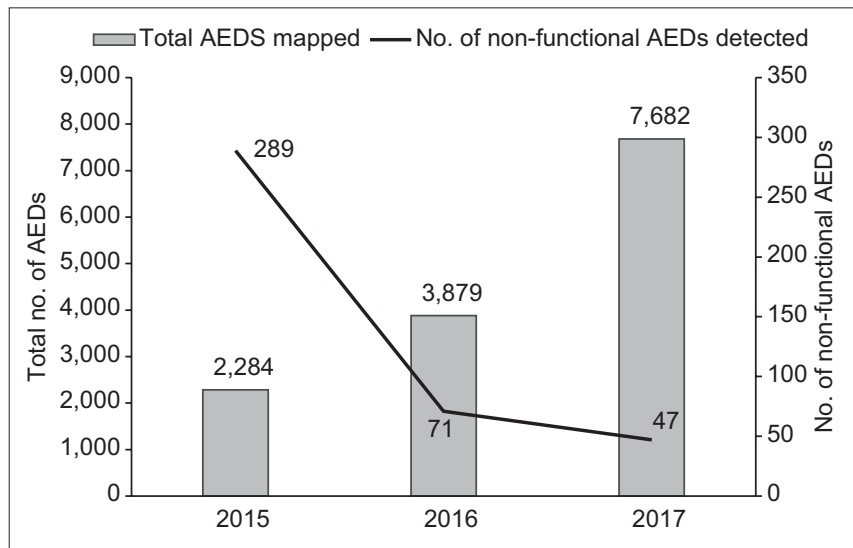

Fig. 1 Chart shows the number of AEDs in the registry and the number of non-functional AEDs detected each year. AED: automated external defibrillator room were unaware of the existence of these AEDs. ${ }^{(12)}$ Due to the absence of servicing contracts, maintenance of the AEDs was performed by the individual organisations, who, due to the low utility, may not be aware of the need for regular battery and pad replacement. In 2014, the MHA started the first AED leasing contract for public access AEDs under the Save-A-Life scheme, which bundled the cost of maintenance with that of replacement batteries and pads. Their upfront cost is much lower compared to individual AED purchases, making AEDs more affordable for building and business owners. Any damage or theft will be handled by the leasing company and regular CPR and AED training can also be provided for designated providers as part of this programme, to maintain currency.

\section{CONTINUOUS EDUCATION}

The local bystander defibrillation rate of $2.1 \%^{(7)}$ pales in comparison to $3.5 \%$ in Taiwan ${ }^{(13)}$ and $3.8 \%$ in Copenhagen, Denmark. ${ }^{(14)}$ According to a local population-based survey conducted in $2009,31.4 \%$ of participants were CPR-trained and $10.7 \%$ were AED-trained. ${ }^{(15)}$ In addition, only $57.2 \%$ believed that they should be trained in AED as compared to $82.6 \%$ for CPR.

To promote the importance of CPR and AED and usage of myResponder during cardiac arrest, the SHF conducted roadshows and used online resources to publish survivors' stories. Various CPR and AED training programmes are available. The DARE (Dispatcher-Assisted first REsponder) programme is a simplified 45-minute session in which participants watch a scenario-based video, then have a hands-on session to learn to dial 995 in an emergency, perform CPR as directed by the dispatcher, and use an AED. The full CPR and AED certification course is available for those who wish to learn more.

Due to limitations in Singapore's existing legislation, several issues are not addressed by R-AEDI. Firstly, the AED registry is

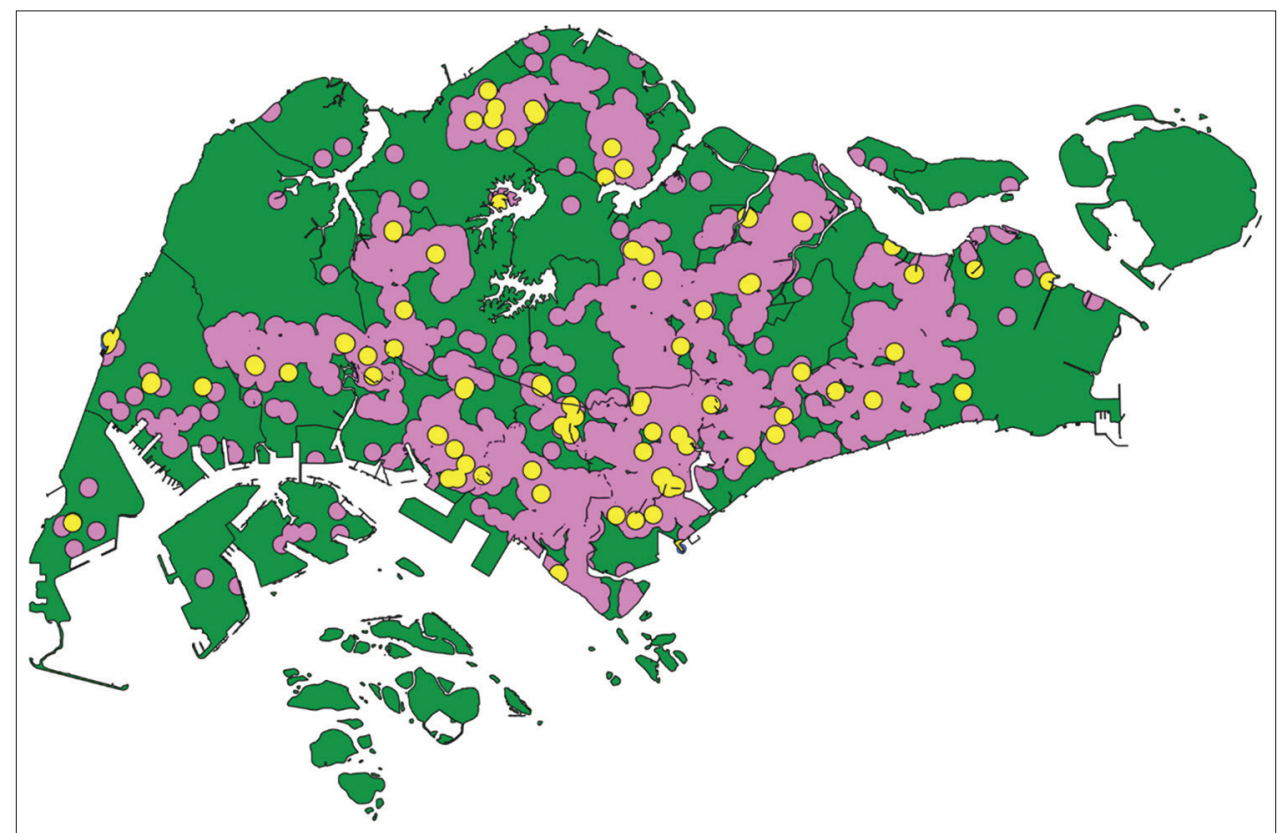

Fig. 2 Map shows the distribution of AEDs in Singapore. Areas that have public access AEDs within $400 \mathrm{~m}$ are highlighted in purple, while the yellow circles represent AEDs meant for private use. AED: automated external defibrillator 
not legally mandated. There is no legal requirement for AED owners to share information about AED locations. AED owners have also expressed concerns related to cost and liability if the public use their AEDs. There is also no requirement for building owners to have AEDs, or to provide CPR and AED training.

The absence of a Good Samaritan law may also deter bystanders from using AEDs. Without AED laws, there are no requirements for high-risk locations to install AEDs. Most AEDs today also record data when activated, and this valuable information helps in understanding the interactions between the OHCA victim and the rescuers. In some states in the United States, evaluation of each $\mathrm{OHCA}$ event was required so as to develop a quality improvement plan. ${ }^{(16)}$ There is great value in being able to retrieve and study this data.

Last but not least, the myResponder app is unable to discern whether the community first responders, who are self-registered, are genuinely trained in CPR and AED. However, the responders are reminded to attend the DARE programme for training and to render assistance only if they are trained.

\section{THE WAY AHEAD}

As mentioned earlier, there are some key barriers to the use of PADs. R-AEDI helps in locating AEDs via myResponder, which has now incorporated AED availability on the map - whether it is available $24 / 7$ or limited to the operating hours of the compound. Future developments may include gamification so that the public can contribute location-based updates for public AEDs. The SHF has also created an AED pads replacement and subsidy programme to encourage AED owners to allow public usage of their AEDs.

Since the mid-1990s, legislatures across the United States began enacting AED-related laws, including Good Samaritan laws, hoping that these would reduce liability and encourage more organisations and individuals to buy or lease AEDs for public access. Such laws now exist in every state and vary in structure, content, complexity and scope of coverage. Locally, it has been encouraging that despite the absence of an AED law and Good Samaritan laws, Singaporeans have been coming forward to assist in the resuscitation of $\mathrm{OHCA}$ cases.

\section{CONCLUSION}

To improve the survival rates of OHCA victims in Singapore, it is imperative that we concentrate on community efforts to encourage primary prevention through leading a healthy lifestyle, early recognition of OHCA patients and prompt institution of life-saving measures. The Singapore Resuscitation and First Aid
Council has set a target of having at least one first responder who is able to initiate early and prompt life-saving first aid in every household in Singapore by 2020. Through the community efforts initiated by the R-AEDI and Save-A-Life projects, it is hoped that bystander CPR and AED use will continue to increase in Singapore.

\section{ACKNOWLEDGEMENTS}

The authors would like to thank CPT Carl Ross de Souza from the Singapore Civil Defence Force as well as Mr Kenneth See, Mr Lim Kiat and the team from the SHF for their contribution towards the publication of this paper.

\section{REFERENCES}

1. Benjamin EJ, Virani SS, Callaway CW, et al; American Heart Association Council on Epidemiology and Prevention Statistics Committee and Stroke Statistics Subcommittee. Heart Diseases and Stroke Statistics-2018 update: a report from the American Heart Association. Circulation 2018; 137:e67-e492.

2. Atwood C, Eisenberg MS, Herlitz J, Rea TD. Incidence of EMS-treated out-ofhospital cardiac arrest in Europe. Resuscitation 2005; 67:75-80.

3. Guidelines 2000 for Cardiopulmonary Resuscitation and Emergency Cardiovascular Care. Part 12: from science to survival: strengthening the chain of survival in every community. The American Heart Association in collaboration with the International Liaison Committee on Resuscitation. Circulation 2000; 102(8 Suppl):1358-70

4. Ong MEH, Perkins GD, Cariou A. Out-of-hospital cardiac arrest: prehospital management. Lancet 2018; 391:980-8.

5. Hallstrom AP, Ornato JP, Weisfeldt M, et al; Public Access Defibrillation Trial Investigators. Public-access defibrillation and survival after out-of-hospital cardiac arrest. N Engl J Med 2004; 351:637-46.

6. Tanaka H, Ong MEH, Siddiqui FJ, et al; PAROS Clinical Research Network. Modifiable factors associated with survival after out-of-hospital cardiac arrest in the Pan-Asian Resuscitation Outcomes Study. Ann Emerg Med 2018; 71:608-17.e15.

7. Harjanto S, Na MX, Hao Y, et al; PAROS study group. A before-after interventional trial of dispatcher-assisted cardio-pulmonary resuscitation for out-of-hospital cardiac arrests in Singapore. Resuscitation 2016; 102:85-93.

8. Smith CM, Lim Choi Keung SN, Khan MO, et al. Barriers and facilitators to public access defibrillation in out-of-hospital cardiac arrest: a systematic review. Eur Heart J Qual Care Clin Outcomes 2017; 3:264-73.

9. Ong ME, Shin SD, De Souza NN, et al; PAROS Clinical Research Network. Outcomes for out-of-hospital cardiac arrests across 7 countries in Asia: the Pan Asian Resuscitation Outcomes Study (PAROS). Resuscitation 2015; 96:100-8.

10. Larsen MP, Eisenberg MS, Cummins RO, Hallstrom AP. Predicting survival from out-of-hospital cardiac arrest: a graphic model. Ann Emerg Med 1993; 22:1652-8

11. Sakai T, Iwami T, Kitamura T, et al. Effectiveness of the new 'Mobile AED Map' to find and retrieve an AED: a randomised controlled trial. Resuscitation 2011; 82:69-73.

12. Zakaria ND, Ong ME, Gan HN, et al; PAROS study group. Implications for public access defibrillation placement by non-traumatic out-of-hospital cardiac arrest occurrence in Singapore. Emerg Med Australas 2014; 26:229-36.

13. Wang TH, Wu HW, Hou PC, Tseng HJ. The utilization of automated external defibrillators in Taiwan. J Formos Med Assoc 2018; 118(1 Pt 1):148-51.

14. Agerskov M, Niselsen AM, Hansen CM, et al. Public access defibrillation: great benefit and potential but infrequently used. Resuscitation 2015; 96:53-8.

15. Ong ME, Quah JL, Ho AF, et al. National population based survey on the prevalence of first aid, cardiopulmonary resuscitation and automated external defibrillator skills in Singapore. Resuscitation 2013; 84:1633-6.

16. Gilchrist S, Schieb L, Mukhtar Q, et al. A summary of public access defibrillation laws, United States, 2010. Prev Chronic Dis 2012; 9:E71. 